\title{
Progress on the Extraction Techniques of Bromine
}

\author{
Fei Ge, Yuting Li ${ }^{*}$, Xiushen Ye, Haining Liu \\ Qinghai Institute of Salt Lakes, Chinese Academy of Sciences, Xining 810008, China \\ * Corresponding Author. E-mail address: yexs@isl.ac.cn.
}

\begin{abstract}
Keywords: bromine; ion-exchange method; membrane separation technique; high gravity air stripping technology.

Abstract: During the past few years, the concentration of bromine in bromine-bearing resource in China is dropping quickly. However, the traditional methods, such as air blow-out technique has the disadvantages of high energy consumption and narrow range of application on temperature, and it is not profitable if the concentration of bromine in feed was becoming lower. In this paper, based on a brief analysis of steaming-out and air blow-out technique, the progress in some novel technologies of bromine extraction including ion exchange resin adsorption, membrane process and high gravity air stripping technique are reviewed. The advantages and further study directions of these technologies are discussed.
\end{abstract}

\section{Introduction}

As the only liquid nonmetal substance at the room temperature, bromine is one of the basic chemical raw materials. The down-stream products are various, such as oil drilling cleaner, flame, refrigerant, water treatment agent and pharmaceutical intermediates and so on.

Bromine primarily exists in seawater, surface and underground brine and salt lake in the form of ionic compound. The bromine content is $65 \mathrm{~g} / \mathrm{m}$ in the sea, $2000 \sim 4800 \mathrm{~g} / \mathrm{m}$ in the underground brine of America, $5000 \mathrm{~g} / \mathrm{m}$ in the Dead Sea brine of Israel, respectively [1]. The most useful raw materials for extracting bromine in China mainly exist in thickening seawater and underground brine after desalinating of the seawater in the districts of around Bohai and Weifang. A number of studies have been devoted to the problem of recovering bromine. Now the productive techniques of extracting bromine mainly take the methods of steaming-out and air blow-out. Among them the bromine produced by steaming-out occupies only $10 \%$ in all country of productive power, by comparison $90 \%$ produced by air blow-out technique.

\section{The techniques of extraction bromine}

\section{A. Steaming-out and air blow-out technique}

Steaming-out technique was the earliest method to extract bromine in chemical industry application. The brine was acidulated and put into packed column where the bromine ion was oxidized by the countercurrent chlorine. Free bromine was brought into the condenser by liquid of water and bromine with different volatility. Then the steam was condensed and pumped out as the product. With the advantages of simple operation and low consumption of raw material, the method was suitable to extract bromine in a large scale. The higher concentration brine was; the less steam consumption was needed $[2,3]$. In order to obtain higher interests, the technique was applied to the fields such as bittern, well bittern, oil and gas bittern. On the other hand, considering the economy of bromine extraction, the temperature of liquid required was so high, meanwhile the hydrolysis of free bromine and the reaction of bromine with excessive chlorine influenced the oxidation and blow-out rate.

With the improvement of requirement, the air blow-out method had been widely used. The principle of air blow-out technique was using air as the carrier of free bromine, and the desorption was operated in a packed tower. The free bromine in brine after acidification-oxidation was blown out into the absorption tower, then the absorbing agents such as sulfite liquor or lye was used to absorb the free bromine which was acquired in the course of desorption process. Thus the goal of bromine enrichment 
was achieved [4]. Many absorbents such as the solution of caustic soda $(\mathrm{NaOH})$ and soda $\left(\mathrm{Na}_{2} \mathrm{CO}_{3}\right)$, sulfur dioxide $\left(\mathrm{SO}_{2}\right)$ and iron $(\mathrm{Fe})$ were used. The technique was the most usual method in the bromine extraction because of the simple process and convenient operation. However, this technique needs large equipment and great energy consumption, and it can be seriously influenced by temperature [5].

\section{B. Ion exchange resin adsorption}

Based on the extraction of iodine, ion exchange resin adsorption began to be used in the field of bromine extraction in the 1960s. First, during chlorination and oxidation bromide ion was changed to free bromide which was absorbed by the ion-exchange resin, then the loaded resins can desorb with $\mathrm{H}_{2} \mathrm{SO}_{3}$ and received bromide ion. At last for eluting the bromine ion which is adsorbed in the resin, the recycling agent just like hydrochloride was used. The eluent was oxidized by chlorine and the steam distillation was applied to obtain the finished product $[6,7]$.

Since the 1970s, a number of experiments had been conducted on ion-exchange resin to increase the bromine extraction effectively. Wang et al. [8] studied on the adsorption property of $201 \times 7,201 \times 4$ and D201 anion exchange resins for bromide ion in dense seawater. The result showed that the $201 \times 7$ resin had a good absorptivity for bromide ion. At 298K, the capacity of static saturation adsorption for bromide ion in seawater was $25.94 \mathrm{mg} / \mathrm{g}$, and the capacity of dynamic saturated adsorption quantity could be obtained $13.42 \mathrm{mg} / \mathrm{g}$ when the flow velocity was $2.3 \mathrm{~mL} / \mathrm{min}$. Through the experiment, sodium citrate was selected as desorption agent and the dynamic adsorption experiments showed that with increasing of the seawater flow rate, the penetration volume and the adsorbing capacities of resins decreased.

Meanwhile, Zhang et al. [9] investigated the thermodynamic behavior of bromine adsorption from concentrated seawater with $201 \times 7$ strong-basic anion-exchange resin and the adsorption isotherm data were measured at various temperatures and the thermodynamic property changes were calculated. The experimental data were well fitted by Langmuir equation. The enthalpy change of the bromine adsorption process was positive, which indicated the process was endothermic. The adsorptive capacity of bromine was $2.489 \mathrm{mg} \mathrm{Br} / \mathrm{mL}$ wet resin and attained the highest adsorption rate of $98 \%$. It suggested $201 \times 7$ resins was the most effective one for bromine adsorption.

Compared with air blow-out, resin adsorption has a series of advantages such as lower energy consumption, less temperature effect and no brine resolve. The adsorption of bromine is independent with the temperature at the range of $0 \sim 60^{\circ} \mathrm{C}$, but there were still many drawbacks affected the industrial application such as the resins required high resistance to cracking, chemical degradation and dissolution. Simultaneously, high steam consumption and the resins were fragile in the process of elution also limited its industrial applications $[6,10]$.

\section{Membrane process}

1). Gas membrane process

The gas membrane separation technique which was applied to bromine extraction from the brine has been studied in the beginning of 1980s. It was applied to bromine extraction from the brine and aqueous solution which contained trace bromide, The basic principle of the technology was that the bromine volatilized into gas between film hole and electrolyte interface when the material fluid through the membrane pores. With the driving force which caused by bromine concentration gradient in the two sides of membrane, the gaseous bromine transited to the other side of the membrane, then the separated gaseous bromine can be adsorbed by the sorbent [11,12]. The main technical characteristic of the gas membrane process was that the bromine content in brine and temperature were the main factors which were directly proportional to flux, yield and mass transfer coefficient.

The gas membrane separation technique has the advantages of less adsorbents using, higher efficiency, no secondary contamination, lower energy consumption which just consumed half of the electric of the technique of air blow-out, simpler equipment and more convenient operation. Many scholars had conducted actively explore in the world. E. L. Cussler [13] used flat polypropylene membranes to research the process of bromine extraction from seawater in the early time.

Yao et al. [14] studied on several types of PVDF hollow fiber membranes and used in the gas membrane separation process for extracting bromine from simulated seawater or concentrated 
seawater. A type of PVDF membrane module was chosen as the course of the experiments and the results suggest that the PVDF membrane fabricated following the preparation method of this one was suitable for extracting bromine from seawater or concentrated seawater. And if it could be washed at regular intervals based on the change of mass transfer coefficient or flux, the membrane could work for a longer time.

Wang et al. [15] used a new type of polymer material which was polyvinylidene fluoride (PVDF) to study the extraction of bromine from seawater and brine. The results showed that increased pressure difference between two sides of the membrane could not improve flux. The impetus of this mass transfer process was not pressure difference, but concentration difference. The efficiency, mass transfer coefficient and flux were proportional to temperature, and the efficiency, mass transfer coefficient were more obvious than the flux. The flux increased greatly with the increased concentration of seawater, but the efficiency and mass transfer coefficient were less affected.

2). Emulsion membrane process

Liquid membrane originated from Dr. Li NN in the late 1960s. Emulsion liquid membrane body was first suggested which was used to separate the organics in hydrocarbons or wastewater [16]. Then the research activities on liquid membrane separation processes in bromine extraction fields had been paid close attention.

Jiang et al. [17] researched the the bromine extraction from seawater by the liquid membrane. The experiments used the polyether FG7921 and kerosene to form the liquid membrane and used different concentration of $\mathrm{Na}_{2} \mathrm{CO}_{3}$ as inner phase to form emulsion liquid membrane system. The research result showed that under the optimum conditions the recovery yield reached over $97 \%$.

Wang et al. [18] studied on bromine extraction from sea water by emulsion liquid membrane. The factors influencing extract performance, such as surfactant concentration, water phase concentration, ratio of emulsion and inner water phase (REW) and ratio of oil phase to inner water phase (ROW) was investigated. The results showed that under the condition of kerosene as solvent, L-113A concentration was $0.54 \%, \mathrm{Na}_{2} \mathrm{CO}_{3}$ as water phase was $0.05 \mathrm{~mol} / \mathrm{L}$, ROW was $1: 1$, REW was $1: 40$, the time of emulsion preparation and extraction were $18 \mathrm{~min}$ and $8 \mathrm{~min}$, respectively. The extraction rate could be reached $99.4 \%$ which suggested that the emulsion liquid membrane technique could effectively extract the bromine from the seawater.

The technique had a number of advantages including the higher efficiency, the better selection and saving energy, and it was especially applied to the separation of the specific ion and organic substances in the solution [19]. Compared with the resin absorption technique, the extraction, cleaning and regeneration of the emulsion liquid membrane technique finished in one step, and the technique also has the advantages of simpler operation, saving energy, less excretion, higher selectivity and so on, whereas many deficiencies were also obvious, such as the bromine extraction yield was influenced by osmotic pressure between the seawater which inside and outside membrane phase and absorbing liquid. The reduced stability of liquid membrane was easy to lead to the break of liquid film then the bromine extraction yield was reduced [20].

\section{High gravity air stripping technique}

Based on the lower stripping rate and higher energy consumption of the sour adsorption way of air stripping, the main technology of air stripping by high gravity is proposed for extracting bromine from brine domestically.

The free bromine in the oxidized liquid was blown out by high gravity separator which used the characteristic of the high strength mass transfer. In the high gravity field, under the gravity of the packing rotating, the liquid which was located in the top of the inlet and after distributed by liquid distributor was cut to the form of drop, liquid membrane, brine and so on which were conveyed from the inside packing to the outside.

Gas-liquid two phase contacted in cross-flow in the packing and the material liquid which was dispersed and crushed to the maximal and continuous renewal surface in the high speed rotors in the reaction of packing, then contacted with air reversely with great relative speed and blown out the free bromine in the crooked channel in the circumstances of high dispersed, high turbulent, strong mixing and fast renewal surface [21]. 
In 2009, Liu et al. [22] explored the effect of gas to liquid volume ratio, $\mathrm{pH}$ value, super gravity factor and total bromine concentration of oxidized liquid on the stripping rate of free bromine in the air stripping process by high gravity. The results showed that the single-stage and three-stage stripping rate of the oxidative liquid which the total bromine mass concentration was $250 \mathrm{mg} / \mathrm{L}$ were about $88 \%$ and $93 \%$ respectively under the operational conditions of temperature was $20-25^{\circ} \mathrm{C}$, gas-liquid volume ratio was $120, \mathrm{pH}$ value was 3.5 , super-gravity factor was 84.67 , which is about $10 \%$ higher than the air stripping rate of $75 \%-85 \%$ in the traditional tower equipment .Under the same operational conditions, the single-stage stripping rate was $94.5 \%$ with the total bromine mass concentration of $2000 \mathrm{mg} / \mathrm{L}$ provided. The stripping effect for bromine was remarkable, and the energy consumption was decreased.

In 2011, Tan et al. [21] independently developed a gravity separator applied to the extraction of bromine. The blow out rate could reach $99.28 \%$ when the temperature was $25^{\circ} \mathrm{C}$, the material solution $\mathrm{pH}$ was 3.5 , the high gravity factor was 143.75 , the gas-liquid volume ratio was 80 and the packing was high density.

\section{Conclusions}

In the paper, the extraction bromine techniques of steaming-out, air blow-out, ion exchange resin adsorption, membrane process and high gravity air stripping were presented. The steaming-out and the air blow-out were relatively mature technologies which have been widely used all over the world. The technique of steaming-out technique was used to extract bromine from high-concentration brine, which and had the characteristic of simple process simplification but also had the problem of high energy- consumption. The air blow-out method was suitable for extracting bromine from low concentration of brine which has the disadvantages of high energy consumption and could not able to work correctly at the lower temperature $\left(\leq 10^{\circ} \mathrm{C}\right)$.

Bromine was one of the important chemical materials and has wide application in the national economy. However it was very shortage in our country. New technologies and new methods for extracting bromine should fully considering conditions of brine resources and bromine content in China and focus on improving the extracting efficiency and reducing the energy consumption. There will be a considerable space of the extracting bromine techniques and with the development of chemical industry this technique will be widely developed.

\section{Acknowledgements}

This work was financially supported by National Natural Science Foundation of China (51403229), the Foundation of Youth Innovation Promotion Association, CAS (Y310031024), Natural Science Foundation of Qinghai Province (2015-ZJ-933Q), and the West Light Foundation of CAS.

\section{References}

[1] Chunying Li, Qi Li: Bromine compounds preparation manual(Chemical industry press, Bejing 2008).

[2] Haimin Li, Huaide Cheng, Quanyou Zhang : Journal of Salt Lake Research Vol .11 (2003), p. 51

[3] Chengchen Hou, Eizhen Sun: Journal of Sea-lake salt and chemical engineering Vol. 19(1989), p. 17

[4] Zhao Wei, Sihong Zhang, Yongjun Cui: Chinese Journal of Chemical Education Vol. 7(2011),p.1

[5] Linna Zhang. Basic studies on process of air stripping by high gravity in technology of extracting bromine from brine abstract[D]. Taiyuan, North university of china, 2009: 5-6.

[6] Changluo Zhu, Jianjun Kou: Multipurpose Utilization of Mineral Resources Vol. 10 (2003),p. 14 
[7] Jilin Yang. Study on air blow-out method for extraction of bromine [D].Tianjin: Tianjin University, 2001:10

[8] Yingxiu Wang, Caifeng Bi, Yu Zhao, Zuo Jian, Aoqiang Sun: Journal of Ocean University of China Vol. 6(2011),p. 81

[9] Nahui Zhang, Junhong Qiu: Journal of ion exchange and adsorption Vol. 27(2011),p. 26

[10] Jinggang Dong, Kuiwu Ji: Sea-Lake Salt and Chemical Industry Vol. 26 (1997),p. 21

[11] Guoqiang Wang, Shufen Zhang, Licong Wang, Ying Yao, Rongfen Zhao: Ocean Technology Vol. 23 (2004),p. 77

[12] Hong Wang. Extrac tion of brom ine from brine by emulsion liquid membrane[D]. Tangshan: North China University of Science and Technology, 2006: 1-11.

[13] Qi Zhang, E L Cussler: J. Membrane. Sci Vol. 24(1985),p. 43

[14] Ying Yao,Wei Liu, Huifeng Zhang, Ronghua Cai,Ying Wang, Shubao Gao, Dan Wu, Yushan Zhang: Adv. Mater. Res. Vol. 217-218(2011),p. 802

[15] Guoqing Wang, Shufen Zhang, Licong Wang, Ying Yao, Rongfen Zhao: Sea-Lake Salt and Chemical Industry Vol. 33 (2004),p.17

[16] Nana Li, U.S. Patent 3,410,794.(1968)

[17] Yuanliang Jiang, Tong Wang, Yi Wang: Journal of Liaoning Normal University(Natural Science Edition) Vol. 20 (1997),p. 43

[18] Huaming Wang, Wenbin Xu, Jiangnan Shen: Zhejiang Chemical Industry Vol. 41 (2010),p. 20

[19] Hong Wang, Junjie Zhang, Hongxia Li: Inorganic Chemicals Industry Vol.38 (2006),p. 18

[20] Jiangjiang Yu, Mianping Zheng, Qiang Wu, Zhen Nie, Lingzhong Bu: Modern Chemical Industry Vol.33 ( 2013),p. 47

[21] Naijun Tan, Guoqiang Wang. Journal of Salt and Chemical Industry Vol.40 (2011),p. 4

[22] Youzhi Liu, Linna Zhang, Yu Li, Weizhou Jiao, Xiangdan Song, Jiangze Han: Journal of Modern chemical industry Vol.29(2009),p. 78 